\title{
Professionalization of creative thinking of doctors
}

\author{
Mergalyas Kashapov* \\ Department of Pedagogics and Pedagogical Psychology, Yaroslavl State Demidov \\ University, 150003 Yaroslavl, Russia
}

\begin{abstract}
The article deals with professionalization peculiarities of creative thinking of doctors depending on their qualification level. Thus, doctors of the highest rank have higher rate of creative attitude towards their trade, doctors of the first rank show high intuition level, whereas doctors without any rank have high imagination. Doctors without any qualifying rank tend to have various components of creative reserve, such as imagination and curiosity. The interrelation between doctors' creative ability level and certain creativity manifestations. The high creativity level among doctors without any rank is due to high level of creative thinking. For doctors of the second rank, it is connected with the high level of imagination, and for doctors of the highest rank, it is due to the high originality level. Proof has been obtained that creative thinking and active doctors do not complain about their patients, because they create a certain meaningful field, providing for productive mutual understanding. One criterion of a gifted doctor is the efficiency of his medical activity, which is closely connected to the clinical thinking as an efficient psychic process. Discovering the unknown, due to this cognitive process, gives birth to important professional and personality formations.
\end{abstract}

Development of pedagogical psychology in the times of various social and economic changes is marked with the desire to meet growing professional requirements. One of the current innovation challenges is the necessity to conjugate educational and professional standards. We should try to increase resourcefulness of professional school graduates in order to decrease the negative impact of certain risks. The resourcefulness is manifested in ability to seek and see resources, make use of and perfect them.

Research of strong sides of professional thinking is impossible without revealing its resource characteristics. The most important resources (significant, strong, structural, relational) allow not only for the subject to survive the stressful situation, but also successfully continue his professional and personality development. Personality resource is a constructive basis allowing for overcoming difficult life situations. Therefore, knowing

\footnotetext{
* Corresponding author: smk007@bk.ru
} 
the subject's resources would help to influence his mobilization abilities in an adequate and goal-directed way.

The research made by N.V.Grishina proved that psychological peculiarities of man that are potential resources in situation of changes are a less significant factor for the person to be ready to change his life situation than his values, which actually reflect his relationship with the world around him [1]. The resource and value approach worked out by V.P.Poznyakov and T.S.Vavakina allows for analyzing the interrelationship and intercommunication of people in order to reveal their resource orientation in interpersonal interaction [2].

For modern creativity psychology, the issue of proportion between creative thinking as a process and creativity as ability is still urgent. According to V.N.Druzhinin, development of creativity as it is leads to deadaptation. He worked out a 4D model of intellectual range, the top of which is formally indicative intellect, which is formed last of all and is responsible for fruitful creative activity. With its development, the scope for a creative personality realization grows as well [3]. The main ask of creativity psychology is to reveal psychic regularities and mechanisms of creative process and creativeness. Our research has proved that professionals possessing necessary knowledge can not only use their standard thinking, but also adapt the treatment of each patient considering his anamnestic peculiarities. Steady interest to creative activity aimed at cognition and transformation contributes to further professionalization of specialists $[4 ; 5]$.

Medical profession has its own peculiarities and presupposes strict algorithms, rules for diagnosing, treatment and remission, which one has to follow. Still, if doctors always use those schemes for diagnosing and curing, they will stop thinking and «seeing» patients' individual peculiarities, which must be considered for exact diagnosing and successful curing. Therefore, for doctors of different qualification level development of creative thinking is essential for optimal use of their acquired knowledge and getting new one, as well as for perfecting their practical skills in order to fulfill their professional duties efficiently.

In the course of joint research with O.A.Skakunova, we tested the hypothesis of a possible connection between doctors' qualification level and their creative thinking $[6,7]$. The following techniques were used: creative personality skills questionnaire (O.A. Shlyapnikova, M.M. Kashapov), «Creativeness» test (N.F.Vishnyakova), «Incomplete Figures» test of E.P.Tottance (adapted by A.N.Voronin), «Polysemantic Words» technique (T.V.Ogorodova, M.M. Kashapov). The sample group was doctors with various levels of qualification (120 people: 30 doctors of no rank, second rank, first rank and highest rank respectively).

We held correlation analysis of creative ability and creativeness level among doctors with no qualification rank and discovered strong correlation between creativeness level and creativeness rate of «Real Self» image, such as: creative thinking $(0.75, p<0.001)$, originality $(0.67, \mathrm{p}<0.001)$. We discovered medium strong correlation between creativeness level and creativeness rate of «Real Self» image, such as: curiosity $(0.55, \mathrm{p}<0.01)$, imagination $(0.54, \mathrm{p}<0.01)$, sense of humour $(0.51, \mathrm{p}<0.01)$; between creativeness level and creative thinking of «Real Self» image $(0.50, \mathrm{p}<0.01)$; between creativeness level and imagining «Real Self» image $(0.49, \mathrm{p}<0.01)$; between creativeness level and creativeness rate of «Real Self» image, such as: curiosity $(0.40, \mathrm{p}<0.05)$, intuition $(0.38, \mathrm{p}<0.05)$, sense of humour $(0.40, \mathrm{p}<0.05)$, creative attitude to profession $(0.38, \mathrm{p}<0.05)$; between creativeness level and association flexibility $(0.43, p<0.05)$; between creativeness level and discrepancy of «Ideal Self» and «Real Self» images according to «Intuition» scale $(0.39$, $\mathrm{p}<0.05)$.

Interpretation of results proves that high level of creativeness among doctors with no qualification rank is due to high level of their creative thinking. Possessing originality is 
seen as a necessary quality in both ideal and real notion among doctors with no qualification rank. In real and ideal evaluation of doctors, high level of creativeness is related to imagination. Connection between creativeness and sense of humour is weaker in ideal notion than in real one. Connection between curiosity and creativeness level is manifested more in real doctors' notion of themselves than in the ideal one. Connection between level of creativity and emotionality, empathy in real notion of themselves among doctors with no qualification rank, as well as desirable creative attitude towards profession, intuition and creative reserve according to «Intuition», "Verbal Associative Flexibility» scales. The discovered fact contains a cognitive resource, which, when realized, can contribute to successful professionalization of thinking among doctors, since emotional sphere is a powerful creativity source. Sthenic emotions can have positive impact on thinking productivity.

In the course of correlation analysis of creativeness level and creativeness rate among doctors of second qualification rank we discovered high correlation between creativeness level and imagination of «Ideal Self» image $(0.66, \mathrm{p}<0.001)$; medium correlation between creativeness level and creativeness rate of «Real Self» image, such as: curiosity $(0.54$, $\mathrm{p}<0.01)$, imagination $(0.50, \mathrm{p}<0.01)$; between creativeness level and creative reserve of creative attitude towards profession $(0.46, \mathrm{p}<0.01)$; weak correlation between creativeness level and creativeness rate of «Real Self» image, such as: creative thinking $(0.38, \mathrm{p}<0.05)$, originality $(0.39, p<0.05)$, sense of humour $(0.43, p<0.05)$; between creativeness level and creativeness rate of «Ideal Self» image, such as: creative thinking $(0.37, \mathrm{p}<0.05)$, originality $(0.44, \mathrm{p}<0.05)$. In the ideal notion of themselves among doctors of second qualification rank, high level of creativity is related to imagination, in real notion this connection is manifested less. High level of creativeness among doctors of second rank presupposes connection with curiosity. Connection between creativeness level and desirable sense of humour is manifested more in ideal than in real notion among doctors of second rank. We observed connection between creativeness level and desirable creative attitude towards profession, as well as creative reserve of creative attitude towards profession. We also discovered connection between creativeness level among doctors of the mentioned rank with creative thinking and originality. Creativeness level is related to verbal fluency.

Correlation analysis of creative abilities level and creativeness rate among doctors of first qualification rank showed rather weak and statistically unreliable connection. Their average work experience being 14 years and their average age being 39-40, it is possible the doctors are going through professional self-actualization crisis [8], that is why we observe unmatched correlation analysis results.

Creativeness level of high qualification rank doctors is related to curiosity. Connection between creativeness level and originality is manifested more in the real notion of high rank doctors, being less manifested in the ideal one. We discovered connection between creativeness level among doctors of high rank with creative thinking, creative attitude towards profession in real and ideal notions.

Developed creative thinking of a doctor contributes to the efficiency of his work: diagnosing, treatment, prevention of his patients' illnesses. Originality helps doctors in dealing with patients, colleagues and management. What is more, it helps to find a special approach to each of them and find unusual decisions in a given situation. All that gives each doctor a chance to become a unique professional possessing his own style. Curiosity is good for perfecting your knowledge and skills, as well as keeping interest towards your profession alongside with obtaining and keeping high level of diagnosing, treatment and prophylaxis of illnesses. We see creative attitude towards profession as one of the signs of professionalism (despite the fact that it is not required while obtaining a qualification rank). Such attitude towards your profession is possible due to a certain intellectual and 
motivational basis. Professional activity of a doctor presupposes communication and interaction with people (colleagues, patients and their relatives), therefore, each doctor should have developed lexical abilities and verbal creativity.

Summing up the obtained empiric results, we can point out that the basic psychological mechanism of professionalization is characterized by transfer from situational type of thinking to suprasituational one. It is this phenomenon, as our research has proved [9-12], that is the basis of creative professional thinking of a subject. Realizing the suprasituational type of thinking, a doctor has an opportunity not to be limited by the situational framework of treatment process, but go beyond cognitive, organizational and time limits of the comprehended and transformed clinical or deontological situation and make use of his personal potential while communicating with his patient.

The level of situativity is implied in comprehending the specific contents of problem issues formulated by subject in the course of solving professional tasks. Various levels of problematics are defined by, firstly, the objective complexity of a situation, secondly, by the specialist's professionalism and, thirdly, by theoretical or practical orientation of his thinking.

Professionals with suprasituational thinking tend to possess high level of self-analysis, activization of their potential and experience and critical evaluation of their own actions. While solving a problem the subject implies not only practical, but also theoretical professional knowledge. Every act of solving a problem is aimed at self-development and creativity. The situation itself becomes the tool helping to achieve not only specific, but also utmost goals of professional activity. The contradiction contained within a situation becomes the basic stimulus for personal self-development, determining the personal ability to turn your own life activity into the subject of practical transformation. That is, professional constantly goes beyond the limits of available, specific moment of his activity into its reflexive and value context.

Suprasituational thinking has the following resources: stereoscopy, objectiveness and predictive value. Going beyond the limits of comprehended situation helps to adequately determine cause and effect, predict consequence and aftermath, which is possible only through realization of certain actions. In that case, there happens transfer from the phenomenological level of analysis to ontological one (determining general regularities and mechanisms). Such transfer enables adequate comprehension of your subjective position, its substantiation and, if necessary, its required correction. Long term planning is manifested in setting tasks aimed at optimal use of resources available.

On the whole, our concept should be seen as a system of an aggregate of generalized empiric results, formulated as theoretical notions of professionalization of thinking as a certain system of cognitive psychic phenomena: qualities and functions of thinking providing for subject's cognition and perfection of his qualities in the context of his professional activity. These characteristics are the value and parameter to define how much the professional thinking of a subject is formed and expressed. One of the values of professionalization of thinking is a phenomenon or event which helps to judge the subject's thinking process. Analysis of a stressor event is especially important, for it allows understanding if the subject would be able to cope with stress or face the crisis and will have to solve spontaneously arising problems. It should be mentioned that problem situations, as an intellectual difficulty condition, limit and consume resources, or increase them through adequate mobilization of human abilities. The research of doctors' creative thinking proved that everything depends on realization of the ability to creative attitude in the course of work.

Conclusions 
1. It has been established that creativity level among doctors of highest rank is higher than that of doctors of second rank or no rank at all; if we look at doctors of first and second rank, the former definitely have higher level of creativity than the latter.

2. Reliable differences have been discovered in creative attitude towards profession as far as doctors of highest rank and no rank think of themselves (higher with those of highest rank); in imagination (higher among doctors with no rank); in realistic and desirable level of imagination among doctors of second and first rank (higher with those of the first rank); in creative attitude towards profession in terms of «real self» among doctors of second and highest rank (better manifested among those of higher rank).

3. Reliable differences have been discovered concerning the index of non-verbal creativity among doctors of first, second ranks and no rank at all (higher among those of first rank); among doctors of highest rank and no rank (higher among the former). The data obtained corresponds with the supposition of connection between non-verbal creativity level and qualification rank of doctors.

4. Reliable and distinct differences have been established concerning verbal creativity level of doctors: among highest rank and no rank doctors in associative and semantic verbal flexibility, among highest and second rank doctors in semantic verbal flexibility (better manifested among doctors of highest rank); among first and second rank doctors in verbal originality (higher with the former); the tendency of reliable differences is obvious while comparing doctors of no rank and second rank concerning their semantic flexibility (higher among the latter); among doctors of no rank and first rank in originality (higher among the latter).

Work carried out with the financial support from RSSF (Russian State Scientific Foundation) (Project No.19-013-00102a)

\section{References}

1. N.V. Grishina, Psychologicheskiye issledovania. 6 (30). (2013) URL: http://psystudy.ru

2. V.P.Poznyakov, T.S. Vavakina, Resource-and-value approach to analyzing social interaction of joint life activity subjects (MosGU Publishers, Moscow, 2014)

3. V.N. Druzhinin, Psychology of peculiarities: Selected works (Institute of Psychology, RAS», Moscow, Publishers 2007)

4. M.M. Kashapov, Psychology of professional pedagogical thinking. (Author's abstract of Doctor of Psychology dissertation, Moscow, 2000)

5. M.M. Kashapov, Psychologiya i shkola, 1, 64-70 (2008)

6. M.M. Kashapov, O.A. Skakunova, Izvestiya IGU. Psychology series. 27, 16-31 (2019)

7. M.M. Kashapov, O.A. Skakunova, The role of a young doctor's creative professional thinking in his interaction with patients (Collected articles: Psychology of man as subject of cognition, communication and activity, Moscow, 2018)

8. E.P. Ilyin, Psychology of adulthood (Piter, St.Petersburg, 2012)

9. M.M. Kashapov, O.N. Slodchuk, Chelovechesky factor: Socialny psycholog. 31, 415-422 (2016)

10. M.M. Kashapov, G.L Shamatonova, A.S. Kashapov, I.V. Otstavnova, Education integration. 21, 683-694 (2017)

11. M.M. Kashapov, Izvestiya IGU. Psychology series. 22, 3-9. (2017) 
12. M.M. Kashapov, I.V. Serafimovich, Y.V. Poshekhonova, Psychology in Russia: State of the Art. 10, 80- 94 (2017) 